\title{
Not in their genes: Phenotypic flexibility, behavioural traditions and cultural evolution in wild bonnet macaques
}

\author{
ANINDYA SINHA \\ ${ }^{\dagger}$ National Institute of Advanced Studies, Indian Institute of Science Campus, Bangalore 560 012, India \\ Indian Association for the Cultivation of Science, 2A-2B Raja S C Mullick Road, Kolkata 700 032, India \\ Nature Conservation Foundation, 3076/5, 4th Cross, Gokulam Park, Mysore 570 002, India \\ ${ }^{\dagger}$ Address for correspondence (Fax, 91-80-23606634; Email, asinha@nias.iisc.ernet.in)
}

\begin{abstract}
Phenotypic flexibility, or the within-genotype, context-dependent, variation in behaviour expressed by single reproductively mature individuals during their lifetimes, often impart a selective advantage to organisms and profoundly influence their survival and reproduction. Another phenomenon apparently not under direct genetic control is behavioural inheritance whereby higher animals are able to acquire information from the behaviour of others by social learning, and, through their own modified behaviour, transmit such information between individuals and across generations. Behavioural information transfer of this nature thus represents another form of inheritance that operates in many animals in tandem with the more basic genetic system. This paper examines the impact that phenotypic flexibility, behavioural inheritance and socially transmitted cultural traditions may have in shaping the structure and dynamics of a primate society - that of the bonnet macaque (Macaca radiata), a primate species endemic to peninsular India. Three principal issues are considered: the role of phenotypic flexibility in shaping social behaviour, the occurrence of individual behavioural traits leading to the establishment of social traditions, and the appearance of cultural evolution amidst such social traditions. Although more prolonged observations are required, these initial findings suggest that phenotypic plasticity, behavioural inheritance and cultural traditions may be much more widespread among primates than have previously been assumed but may have escaped attention due to a preoccupation with genetic inheritance in zoological thinking.
\end{abstract}

[Sinha A 2005 Not in their genes: Phenotypic flexibility, behavioural traditions and cultural evolution in wild bonnet macaques; J. Biosci. 30 51-64]

Genes code for proteins, not monkeys

-inspired by Bateson 2003

\section{Phenotypic flexibility}

Phenotypic plasticity, which refers to the phenotypic variability expressed by single genotypes often as a func-

This paper is dedicated with admiration and gratitude to the late Dr Raja Ramanna, a man who held all primates in great esteem. tion of environmental conditions varying predictably or in response to more stochastic fluctuations in the environment, is known to strongly influence biological diversity (West-Eberhard 1989), organismal design (Piersma and Drent 2003), environmental adaptability (Travis 1994; Via 1995), and evolution (Gordon 1992; Travis et al 1999) in a wide spectrum of organisms (Rollo 1994; Sultan 1995; Reznick and Travis 1996; Pigliucci 2001). The most well-studied of these is developmental plasticity, the irreversible variation in traits of individuals that results from processes during development as a consequence of environmental variation, and one that is best described by empirical relationships known as reaction

Keywords. Behavioural transmission; bonnet macaque; cultural evolution; Macaca radiata; phenotypic flexibility; social traditions 
norms (Stearns 1989; Schlichting and Pigliucci 1998). Best studied among these is polyphenism, which refers to the ability of certain arthropods and other invertebrates to produce a sequence of generations with different discrete phenotypes to accommodate seasonal changes in the environment (Mayr 1963, 1969; Shapiro 1976; Danks 1999). All these forms of developmental variation are, however, ultimately likely to be under direct genetic control since they are always species-specific and relatively invariant in their manifestation.

Another form of context-dependent variation in behaviour-now referred to as phenotypic flexibility, instead of phenotypic plasticity - includes reversible phenotypic transformations shown by organisms in response to variations in ecological and social environments (reviewed in Piersma and Drent 2003). Flexibility is the choice of term here not only to bring more clarity in defining these phenomena, but also to reflect the fact that such phenotypic variation, which is unlikely to be under direct, rigid genetic control as are developmental variations such as polyphenism, may be displayed by a single individual (which is obviously an advantage in terms of individual fitness) and is usually reversible in nature. It should also be recognized that, in the long term, such variation may represent or may have the potential to become integral aspects of the life-histories of such organisms, and therefore, be subject to natural selection subsequently.

Phenotypic flexibility is most likely to arise in environments where changes occur over time-scales shorter than the lifetimes of individuals; such changes should also be continuous and reversible, allowing individuals to adapt favourably to the prevailing environmental regime and imparting to them a selective advantage (Padilla and Adolph 1996; Kingsolver and Huey 1998). Most studies of phenotypic flexibility have, however, focussed on predictable environments and have largely investigated morphological and physiological variation in plants and animals in response to fluctuations in their physical environment (Piersma and Drent 2003, but see Montgomerie 2001).

Primates, in contrast, live in social environments that often change unpredictably due to efflux or influx of new individuals, or due to sudden changes in environmental factors such as food availability and distribution, which, in turn, significantly affect troop composition and social structure. Are individual primates capable of exhibiting behavioural flexibility under these circumstances? And, if indeed they do adapt behaviourally to such changes in their society, does such flexibility impact their survival and reproduction?

\section{Behavioural flexibility in bonnet macaques}

The bonnet macaque (Macaca radiata, Geoffroy), a cercopithecine primate endemic to but widely distributed in peninsular India, appears to be unusual in exhibiting remarkable social flexibility and a wide variety of behavioural strategies, all of which enable it to adapt successfully to very different ecological habitats (Sinha 2001). The species usually lives in large multimale multifemale troops in which adult individuals develop strong affiliative relationships with each other and adult males display remarkable tolerance of the reproductive efforts and acts of other adult and subadult males.

In recent years, however, one particular population of bonnet macaques (Population I in table 1, described below) - in the dry deciduous forests of Bandipur National Park and Mudumalai Wildlife Sanctuary in southern India - appears to have evolved a new form of social organization in which small troops are formed by a single adult male with a few adult females and their offspring (Sinha et al 2003). These unimale troops are strikingly different from the typical multimale troops not only in their demographic features but also in the nature of the social relationships that develop between the single male and the resident females within the troop (Sinha et al 2005).

The evolution of unimale social organization in this bonnet macaque population appears to be related to changes in food abundance and distribution in its habitat, particularly during summer, and the consequent formation of small groups of matrilineal females that single males can reproductively monopolise (figure 1; Sinha et al 2005). This reproductive monopolization, in turn, is related to a strong intolerance that the single male develops towards other males, both within and outside the group. As prevails in most unimale harem systems (Smuts et al 1987), there are several behavioural manifestations of this intolerance, each of which appears to be unique to unimale troops but virtually never encountered within multimale troops in any population (figure 1). Some of these unusual behaviours include the herding of troop females, severe aggression towards subadult and juvenile males, active involvement in troop defense, and the prevention of male immigration into the troop.

Such despotic behaviour of the resident male in unimale troops leading to increased male emigration from such groups may, in turn, result in important demographic consequences including the observed characteristic depletion of subadult males and the skewing of the birth sex ratio towards female infants in unimale troops (figure 1; Sinha et al 2003). As male emigration at a relatively young age could potentially entail heavy costs, females in unimale bonnet macaque troops may have a selective advantage if they bore and raised daughters, who would remain in the troop and contribute to the matriline, rather than sons, who would almost invariably be driven away. Alternatively, such skewing of the birth sex ratio could 
be mediated by the adult male who would clearly benefit from daughters rather than sons, as the latter could subsequently become his potential competitors for mating opportunities within the troop.

What leads certain bonnet macaque males solitarily to take over small groups of females and enjoy reproductive monopolization over them? One possible answer to this question comes from the observation that several unimale troops in Bandipur-Mudumalai have subsequently become multimale with one to several individuals joining each of them. Preliminary behavioural observations on these troops have revealed that some of the resident males (of the earlier unimale troops) have, at least partially, adopted the behavioural strategies appropriate to a multimale social organization. Such males may have actually been able to achieve this through phenotypic flexibility, by appropriately adapting their behaviour to changes in their social environment independent of any direct genotypic influence.

Interestingly, only a small subset of adult males in this population appear to be capable of displaying such behavioural flexibility and moreover, such differential behavioural strategies - as prevails in unimale and multimale social organizations - have become apparent in the population only over a relatively short time-period of about ten years. It is, therefore, more likely that the observed patterns reflect examples of phenotypic flexibility rather than any underlying genetic change since, in addition, it is difficult to conceive how such unpredictable short-term changes in behavioural strategies within the same individual could be mediated by any kind of direct genetic control. Stronger support for such behavioural flexibility will finally come from long-term observations when the life-history strategies of particular males in

Table 1. Demography, behavioural traits and ethnographic patterns displayed by the study troops of bonnet macaques.

\begin{tabular}{|c|c|c|c|c|c|c|c|c|c|c|c|c|c|}
\hline \multirow[b]{2}{*}{ Troop } & \multirow{2}{*}{$\begin{array}{l}\text { Troop } \\
\text { size* }\end{array}$} & \multicolumn{2}{|c|}{ Adults* } & \multicolumn{5}{|c|}{ Rapid spread of novel behaviour } & \multicolumn{2}{|c|}{$\begin{array}{l}\text { Parent-offspring } \\
\text { similarity }\end{array}$} & \multicolumn{3}{|c|}{$\begin{array}{l}\text { Group-specific } \\
\text { behaviour }\end{array}$} \\
\hline & & Males & Females & I & II & III & IV & $\mathrm{V}$ & VI & VII & VIII & IX & $\mathrm{X}$ \\
\hline \multicolumn{14}{|c|}{ Population I } \\
\hline BM1 & $32 \cdot 2$ & $6 \cdot 2$ & $8 \cdot 7$ & & & & & & & & & & \\
\hline BM2 & $19 \cdot 3$ & $4 \cdot 0$ & $5 \cdot 5$ & & & & & & & & & & \\
\hline BM3 & $4 \cdot 9$ & $1 \cdot 0$ & $2 \cdot 0$ & & & & & & & & & & \\
\hline BM4 & $15 \cdot 5$ & $3 \cdot 3$ & $4 \cdot 4$ & & & & & & & & & & \\
\hline BM5 & $15 \cdot 9$ & 1.7 & $4 \cdot 4$ & & & & 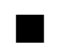 & & $\square$ & & & & \\
\hline BM6 & $31 \cdot 9$ & $6 \cdot 9$ & $7 \cdot 1$ & & & & & & & & & & \\
\hline BM7 & $13 \cdot 6$ & $2 \cdot 5$ & $2 \cdot 4$ & & & & & & & & & & \\
\hline BM8 & $6 \cdot 8$ & $1 \cdot 7$ & $1 \cdot 8$ & & & & & & & & & & \\
\hline BM9 & $20 \cdot 5$ & $3 \cdot 2$ & $5 \cdot 0$ & & & & & & & & & & \\
\hline BM10 & $9 \cdot 8$ & $1 \cdot 0$ & $2 \cdot 3$ & & & & & & & & & & \\
\hline BM11 & $9 \cdot 7$ & $1 \cdot 9$ & $2 \cdot 5$ & & & $\mathbf{\square}$ & & & & & & & \\
\hline BM12 & $20 \cdot 6$ & $3 \cdot 9$ & $5 \cdot 1$ & & & & & & & & & & \\
\hline BM13 & $7 \cdot 4$ & $1 \cdot 3$ & 1.9 & & & & & & & & & & \\
\hline BM14 & $6 \cdot 3$ & $1 \cdot 2$ & $1 \cdot 2$ & & & & & & & & & & \\
\hline BM15 & $27 \cdot 0$ & $6 \cdot 0$ & $8 \cdot 1$ & & & & & & & & & & \\
\hline BM16 & $28 \cdot 6$ & $3 \cdot 5$ & $8 \cdot 0$ & & & & & & & & & & \\
\hline BM17 & $26 \cdot 3$ & $5 \cdot 3$ & $7 \cdot 6$ & & & & & & & & & & \\
\hline BM18 & $12 \cdot 6$ & $2 \cdot 0$ & $3 \cdot 0$ & & & & & & & & & & \\
\hline BM19 & $9 \cdot 8$ & 1.0 & $3 \cdot 0$ & & & & & & & & & & \\
\hline BM20 & $15 \cdot 8$ & $7 \cdot 7$ & 4.9 & & & & & & & & & & \\
\hline BM21 & $5 \cdot 0$ & $5 \cdot 0$ & $0 \cdot 0$ & & & & & & & & & & \\
\hline \multicolumn{14}{|c|}{ Population II } \\
\hline GK1 & $48 \cdot 0$ & $9 \cdot 5$ & $10 \cdot 5$ & & & & & & & $\square$ & & & \\
\hline GK2 & $32 \cdot 5$ & $6 \cdot 0$ & $10 \cdot 0$ & & & & & & & & & & \\
\hline
\end{tabular}

*Each cell shows the mean troop size or the mean number of adult males and females within each population during their respective observation periods.

I, Mango washing; II, bipedal begging; III, car raiding; IV, male adoption; V, tool manufacture; VI, coo-call begging; VII, interaction with humans; VIII, foraging style; IX, branch shaking; X, female affiliative relationships. 
unimale and multimale troops will be known more completely.

Phenotypic flexibility is also shown by bonnet macaque females in the Bandipur-Mudumalai population in different ecological and social environments. Adult females of different dominance ranks, for example, significantly changed their behavioural strategies as they regularly alternated between bouts of natural foraging and feeding on provisioned foods, often during the course of the same day (figure 1; Ram et al 2003). Provisioning was marked by a sharp increase in feeding competition, accompanied by severe aggression and feeding supplants, but individual females were also able to adopt appropriate strategies aimed at reducing social tension within the group. Yet another recent example of behavioural flexibility is that displayed by several adult, subadult and juvenile females in unimale troops, who, faced with a lack of mate choice, have begun to emigrate to other neighbouring unimale or multimale troops, either singly or in small associations (figure 1; Sinha et al 2005).

\section{Changing regimes of food abundance and distribution}

$\begin{array}{ll}\downarrow & \mathfrak{b} \\ \text { Flexibility in female behavioural strategies } & \sqrt{ } \\ \text { dependent on dominance rank } & \sqrt{ } \\ & \sqrt{ } \\ & \sqrt{ } \\ & \mathfrak{b}\end{array}$

Variation in female group size in different ecological contexts

$\downarrow$

\section{Flexibility in male behavioural strategies in unimale and multimale troops

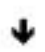 \\ Flexibility in female mate-choice strategies in unimale troops}

Figure 1. A framework for the expression of phenotypic flexibility among bonnet macaques in the Bandipur National Park-Mudumalai Wildlife Sanctuary in southern India. Flexibility in different male and female behavioural strategies (in italics) may have appeared in response to specific socioecological contexts (in normal text) that vary unpredictably over space and time.
The interactions between the individual genotype and environmental components that can generate such behavioural flexibility need to be elucidated and understood. It may, however, be informative to speculate whether the social dynamics that prevail in Bandipur-Mudumalai could ever select for the canalization of each of these behavioural strategies in subsets of individuals that find themselves trapped in certain social or ecological contexts (see Nanjundiah 2003). The answer is probably in the negative unless environmental conditions lead to segregation of these groups and effectively block movement of individuals across them - thus leading to the generation of a stable, predictable social environment. What is clear, nevertheless, is that social lability and individual behavioural variability of this nature may enable a species to evolve novel social and demographic structures as well as innovative behavioural strategies that promote more effective survival and reproduction under periodically changing, but challenging, socioecological situations.

Although interesting in its own right as an example of phenotypic flexibility free of direct genetic determinism, an interesting question is whether such behavioural flexibility can be horizontally or vertically transmitted to other individuals by social learning leading to the establishment of distinctive behavioural traditions, a phenomenon that I discuss below. It is also possible that the adaptability of flexible behavioural strategies to different socioecological contexts may lead to and select for divergent life-history patterns across groups, with the typically extended adult-juvenile contact periods providing opportunities for more cultural transmission (see, for example, Rendell and Whitehead 2001). Our long-term observations on the behavioural profiles and life-history strategies of younger individuals, including offspring, born and raised in these two kinds of social organizations should enable us to examine this possibility in the future.

\section{Lamarckian inheritance systems}

Much attention has been paid, in recent years, to evolved Lamarckian inheritance systems, which, in a sense, signify the evolution of new evolutionary rules themselves (reviewed in Jablonka et al 1998). Notwithstanding the importance of genetic inheritance in governing evolution, different kinds of information-transmission systems appear to have evolved several times during the history of life and these have facilitated the inheritance of acquired characters across generations. Such characters, of different types and occurring at different levels of organization molecular, cellular or organismic - are usually acquired by these biological systems through interactions with their environment, affect the structure and function of the system that acquires them and are often retained for 
transfer to the next generation (Ho and Saunders 1984; Jablonka and Lamb 1989, 1998; Jablonka and Szathmáry 1995; Jablonka et al 1998; Rakyan et al 2001). Notable examples of such Lamarckian inheritance systems include adaptive mutational systems involving non-random changes in DNA, cellular heredity systems involving information transfer through intracellular structures and biochemical mechanisms, behavioural inheritance through social learning within social organizations, and symbolic language systems. These complex mechanisms are believed to have arisen through randomly-generated variations that were subjected to natural selection in environments characterized by re-occurring features, but with enough spatial and temporal variability to preclude a fixed genetic response. Although different from each other in the type of information they transmit, in their evolutionary history and in their evolutionary effects, such systems also clearly interact - and it is the characterization of such interactions that poses a great challenge to evolutionary biology in the future.

\section{Behavioural inheritance and social learning}

Animals acquire various kinds of information during their interactions with the physical and social environment, which is stored in their nervous system. Such information, usually in the form of patterns of behaviour, can be transferred between individuals and across generations (see Avital and Jablonka 2000). Variation in such socially acquired and transmitted behaviours is unlikely to be under direct genetic control since individuals who are closely related genetically can have and pass on very different behaviours; this is also true for cultural traditions that such behaviours may generate. New behavioural patterns, first acquired by accident or by individual learning in a new environment is usually transferred to other individuals by social learning. This form of inheritance occurs at the level of the organism and the information encoded is analog in nature - that is, continuous and not readily dissociable into constituent heritable parts. The information is contained, in fact, within the dynamics of interaction between the individual and its social and ecological environment, and when transmitted to another individual, this dynamic system may be reconstituted once again in the new bearer of the pattern.

In birds and mammals, social learning occurs when the presence of one relatively experienced individual increases the chances that a naïve individual will learn a similar behavioural pattern. There are various mechanisms of social learning that differ from each other in levels of complexity and degree of involvement of the participating individuals with each other (Whiten 2000), but the three most significant categories are imitation, emulation and stimulus enhancement. Imitation occurs when one individual learns some part of the form of a behaviour from another individual (Whiten 2000), while emulation has been defined differently as either the recognition by one individual of what a tool can afford to achieve while observing another individual use it for a definite purpose (Tomasello et al 1987; Tomasello 1996) or the recognition by one individual of the goal that is to be pursued while observing the behaviour of another individual (goal emulation, Whiten and Ham 1992). Stimulus enhancement, on the other hand, is when one individual learns from another to what object or location it should orient its behaviour (Whiten 2000).

Social learning may often remain restricted to motheroffspring pairs or remain constrained within close genetic relatives who preferentially associate with each other, as will be discussed later, but in many cases, may spread through a particular population to become a behavioural tradition, characteristic of the group. Although such traditions (from the Latin root meaning 'handing over') have often been termed culture, this has been contested by various authors who argue that culture can only come into existence through true imitation and others who are of the firm opinion (although highly debated) that true imitation has virtually not yet been demonstrated in any nonhuman animal (Galef 1992; Tomasello et al 1993; Kuper 1999; Rendell and Whitehead 2001; Laland and Hoppit 2003; see also Byrne et al 2004). I will, however, continue to use the terms 'tradition' and 'culture' for such behavioural patterns that diffuse across a group of individuals through some form of social learning and is subsequently displayed by a significant membership of the group (see also Galef 1992).

There are a number of well-known examples of such behavioural traditions in animals that have been transmitted within and across generations such as opening of milk caps by British birds (Fisher and Hinde 1950, 1952), washing of sweet potatoes and wheat by Japanese macaques (Kawamura 1959; Kawai 1965; Kawai et al 1992) or the elegant example of pine cone-stripping by Israeli black rats (Aisner and Terkel 1992; Terkel 1996). When a population of these rats extended their foraging range into forests of the Jerusalem-pine, they were forced to feed on the edible seeds of this pine but only after they had devised a sophisticated technique of stripping of the tough, inedible cone in which these seeds were embedded. This behavioural technique soon spread across entire populations using these forests; cross-fostering experiments where non-stripping pups were raised by stripping mothers and vice versa showed that the ability to strip pine cones efficiently is socially learnt and not genetically determined. Since then, a number of elaborate case studies of social learning and behavioural traditions in animals have been established; besides the well-documented cultures 
of chimpanzees (Tomasello 1990; Whiten et al 1999) and orangutans (van Schaik et al 2003), these include instances of behavioural transmission (occasionally leading to social traditions) as diverse as mate choice in guppies (Dugatkin 1996), vocalisations in ravens (Enggist-Dueblin and Pfister 2002), feeding techniques of Tonkean macaques (Drapier and Thierry 2002), abnormal behaviours of captive rhesus macaques (Hook et al 2002) or prey capture by killer whales (Rendell and Whitehead 2001).

\section{Behavioural traits and social traditions in bonnet macaques}

Most behavioural traditions clearly begin from an individual behavioural trait - that either arises by accident or by individual learning when an animal is confronted with a novel environment or a new environmental stimulus. Importantly, this process itself serves in the generation of individuality and leads to an increase in behavioural variation within the group in which such novel individual traits arise. In fact, it has been argued that the evolution of Lamarckian processes of transmission of acquired characters needs to be understood if one is to obtain insights into the transition of life-forms to new levels of individuality (Jablonka 1994; Jablonka and Szathmáry 1995; Maynard Smith and Szathmáry 1995).

Do social traditions exist in wild bonnet macaques? I present below some preliminary findings on possible instances of behavioural transmission observed in two well-separated populations of bonnet macaques over more than a decade (1993-2004). It must be stressed here that my field observations on individual behavioural traits and social traditions in these macaques have followed a system akin to ethnographic methods in anthropologyinvolving primarily the documentation of differences in individual behavioural repertoire and practices across groups and populations. It should be noted that a raging controversy exists over whether such documentation alone is enough to establish the presence of animal culture or whether a rigorous demonstration of social learning and transmission of behaviours is also essential to the process (Heyes and Galef 1996; see also Rendell and Whitehead 2001 and peer-reviews therein). No attempts have, however, been made in this study to observe behavioural transmission in the laboratory and it has also never been practically possible in the field to unravel the exact mechanisms of social learning that may have mediated the spread of particular behavioural practices, wherever these have been observed.

Behavioural transmission leading to a social tradition has been deduced from spatial, temporal or social patterns of variation in behaviour that were not consistent with genetic or environmental determination, and did not appear to have arisen by individual learning. Again, no attempts were made to deduce what particular form of social learning was responsible for the observed patterns. In general, three types of such patterns have been considered (Cavalli-Sforza and Feldman 1981):

(i) Rapid-spread patterns: Rapid spread of a novel form of behaviour (that arises in an individual rather than as a generalized response by several individuals) through a segment of the troop or population, indicating a largely horizontal, within-generation cultural process.

(ii) Parent-offspring patterns: Similarities in novel complex behaviour between mother and offspring, indicating a vertical, parent-offspring transmission.

(iii) Group-specific patterns: Differences in behavioural patterns between stable groups of individuals that may be difficult to explain in terms of genetic differences, shared environments, or the demographic sizes and structures of the group. Although often not clear, such patterns could arise through a combination of vertical or oblique transfer within matrilineal groups or by vertical, oblique and horizontal transfer with individuals aligning their behaviour with that of other group members (Boyd and Richerson 1985) in more labile groups.

A point that should be noted here is that the above categorization is not discrete since mother-offspring patterns could contribute to group-specific patterns as could rapid-spread patterns. Attention should, however, be paid to the exact mode of transmission, wherever possible, since, in contrast to within-generation transfer of patterns (as usually occurs in rapid-spread patterns), across-generation transmission (in mother-offspring patterns and more commonly in group-specific patterns) is likely to have more profound evolutionary effects in the long term (Russell and Russell 1990; Feldman and Laland 1996).

The present analysis is based on preliminary data acquired through behavioural observations on two populations of bonnet macaques, which have been studied for different periods of time over the last eleven years, beginning March 1993.

Population I primarily consists of 21 troops (Troops BM1 to BM21; table 1) inhabiting the south-eastern region of Bandipur National Park, Karnataka state, and a small adjoining section of the contiguous Mudumalai Wildlife Sanctuary, Tamil Nadu state, in southern India $\left(11^{\circ} 57^{\prime}-\right.$ $\left.11^{\circ} 76^{\prime} \mathrm{N}, 76^{\circ} 55^{\prime}-76^{\circ} 68^{\prime} \mathrm{E}\right)$. Although a range of tropical forest vegetation types - from moist deciduous forest through semi-evergreen forest to dry evergreen forest occur within these sanctuaries, the study groups largely inhabited areas with predominantly dry and moist deciduous vegetation. Behavioural observations ad libitum were conducted for a period of over $1000 \mathrm{~h}$ on all adults, subadults and juveniles of the study troops during the period from March 2000 to August 2003. 
Population II consists of two troops - troops GK1 and GK2 (table 1) - inhabiting $1 \mathrm{~km}^{2}$ of dry deciduous scrubland and mixed forests in the GKVK campus of the University of Agricultural Sciences in Bangalore city (c. $\left.12^{\circ} 98^{\prime} \mathrm{N}, 77^{\circ} 58^{\prime} \mathrm{E}\right)$, Karnataka state. A long-term observational study, consisting of over $1200 \mathrm{~h}$ of observation, was conducted on the behavioural ecology of these two partially provisioned troops, occupying adjacent overlapping home ranges, continuously during the period from March 1993 to September 1995. Behavioural data, used for this analysis, are based on approximately $789.5 \mathrm{~h}$ of focal animal sampling on the adult and subadult males $(n=19)$ and females $(n=21)$ in the two troops with a mean $( \pm$ SE) of $18.33( \pm 1.75) \mathrm{h}$ and 21.01 $( \pm 1.13) \mathrm{h}$ of sampling per male and female respectively. Each focal sample of an individual, chosen randomly without replacement, had a duration of $15 \mathrm{~min}$. The performance of group-specific behaviours have been measured either in terms of bouts/h/individual (foraging style), where a bout represents a finite duration of time during which a number of foraging acts were performed, or directly as events/ $\mathrm{h} /$ individual (branch shaking).

\subsection{Rapid spread of novel behaviour}

Certain novel behaviours have been documented to arise in a particular bonnet macaque troop and then spread across individuals within the group over time scales of less than a generation. Although our short-term observations of these behavioural innovations and their spread must be considered preliminary, genetic causation can clearly be excluded in all these cases. Such behaviours are very likely to have spread rapidly by social learning; it is difficult, however, as mentioned above, to observe processes of social transmission in the field. It is also necessary to rule out the possibility that the different individuals displaying these behaviours could have learnt them independently under the same environmental regime. A brief description of these behavioural innovations are given here, while the troops in which these have been observed has been listed in table 1 .

5.1a Mango washing: Two individuals in an all-male bachelor troop (troop BM21) were observed to collect wild mangoes (Mangifera indica) that had floated down the river Moyar in the Mudumalai Wildlife Sanctuary and wash them by repeated dipping in water followed by vigorous rubbing with their palms before ingestion. Washing could have been performed to remove the slime layer and/or the sticky sap that covered these fruits (pers. obs.) although the fact that washing was also carried out while the fruit was being eaten suggests that it could have also served to soften the hard endocarp of the seed. On one occasion, an individual repeatedly lost the fruit, which he was eating, in a mass of floating vegetation and was observed to vigorously wash it each time that it was recovered.

5.1b Bipedal begging: This behaviour involves the systematic begging for food, displayed by three adult individuals - two males and one female - of troop BM4, while standing erect bipedally on their hindlegs. Although a few individuals belonging to different troops in Population I were randomly observed to beg food in this manner from tourists visiting the Bandipur National Park and Mudumalai Wildlife Sanctuary, these were never performed repeatedly or did not last prolonged periods of time, as was shown by the troop BM4 individuals. It is also noteworthy that the three individuals who systematically displayed this behaviour were the highest-ranked among the males and females in that troop respectively and those least shy when interacting with humans. This implied a possible underlying role for temperament in this behavioural innovation - only individuals that were motivated strongly to approach people and beg food from them were perhaps able to learn this behaviour from one another or were inclined to display it. Several other troops in this population - particularly around human habitations - also had individuals who were not shy in begging for food, but these individuals never displayed this behaviour, suggesting that social learning could have been responsible for the spread of this behaviour in troop BM4.

5.1c Car raiding: This behaviour, displayed sporadically only by very few adult males in Population I, involves the entering and raiding of tourist vehicles for human foods kept in them. The alpha male (named BS) of troop BM11 regularly displayed this behaviour, although no other individual in his troop, including a juvenile male, initially did so. Approximately a year later, the juvenile male began to show car raiding after he had sexually matured into a subadult. Finally, another adult male (named WS), which had immigrated into this troop, began to display car raiding, but only three months after he had joined BS and the subadult male.

5.1d Male adoption: A large juvenile male (named TH) in troop BM5 in the Bandipur National Park exhibited an unusual behaviour in August 2002 when he 'adopted' an orphan six-month-old male infant-continuously carrying him ventro-ventrally, repeatedly allogrooming him and protecting him from aggression shown by other troop members. Such carrying and apparent adoption of an orphan infant by a juvenile or adult male has never before been reported in the primatological literature. Subsequently, another large juvenile male (named DA) began to 
carry the infant as well, though not to the extent that $\mathrm{TH}$ did. These affiliative interactions between TH, DA and the male infant continued till April 2003 (although the infant was not actively carried by TH or DA after November 2002) after which the infant was not seen again and presumed dead. In April 2003, however, two small juveniles - a male and a female - were repeatedly observed to carry a small infant each over short distances; such carrying of infants by small juveniles have never been seen in any other bonnet macaque troop in either of the two study populations.

5.1e Tool manufacture: An adult female (named RI) of troop GK1 in Population I was repeatedly observed to insert a dry stick, stiff leaf or grass blade, or a leaf-midrib into her vagina and scratch vigorously, in response to some irritation that appeared to bother her persistently during the period from March 1993 to September 1994 (Sinha 1997). No other individual in the troop was, however, seen to learn this behaviour, or manufacture and use a similar tool in any other context.

An unusual feature of some of these behavioural innovations - mango-washing, but more specifically, tool manufacture and use - is the specificity of the context in which they were performed. Kummer and Goodall (1985) have, in fact, questioned the importance of such behaviours to the performers themselves and have speculated on the factors that would constrain the transmission of such use in wild populations. The all-male troop (troop BM21), for example, subsequently joined another troop (BM15) and did not spend time along the river Moyar any more; this may have prevented the potential spread of mango-washing to the other troop members. In this case of tool manufacture, it could be argued that such object use may not spread simply because of the narrow window of its applicability.

\subsection{Parent-offspring similarity}

The sharing of certain behavioural traits between mother and offspring, observed in troop GK1, suggests vertical cultural transmission through social learning, although genetic determination or shared environments leading to parallel individual learning could also be potential explanations. The limitations of this study on the bonnet macaques include the failure to monitor these traits over more than two generations and the inability to detect social learning mechanisms that could be potentially involved. Two behavioural traits in this category that were observed are listed here (see also table 1).

5.2a Coo-call begging: Coo calls are usually emitted by adult female and juvenile bonnet macaques when they are lost or separated from their troop (unpublished results). One particular adult female (named SU) in troop GK1 (of Population II) was observed (June 1993 onwards) to employ this call in an unusual situation - when she begged for food in close proximity to humans. Although no other female in this troop ever used this call in a similar situation initially, her two-year-old daughter (named BO) began to use this vocalization in an identical context from November 1994 onwards. Persistent coo call begging was again recently observed among two adult females (possibly an aunt and her niece - named TI and ND, respectively) in troop BM5 (of Population I) - a troop that again occurs in close proximity to human habitation. Evidence in support for the potential of this behavioural innovation to be socially learnt and used by younger females await our future observations on these troops.

5.2b Interaction with humans: Although many bonnet macaque troops live in close association with humans, virtually all adult and juvenile individuals fail to display any kind of affiliative interactions with people. Notable exceptions in this regard were four juveniles - two males (named MI and DO) and two females (named BO and $\mathrm{SH}$ ) - of troop GK1 (in Population II), who regularly interacted with and displayed contact affiliative behaviours with human observers. Remarkably, all these individuals, ranging in age from one to four years, were offspring of a single adult female (named SU). SU was unique among the 11 adult females of this troop in her high degree of tolerance of human observers and in her preferentially close association and extensive affiliative interactions with the adult males of the troop (unpublished results). SU was also unusual in displaying high levels of play behaviour with all the four infants - no other adult female of this troop were ever observed to play with their respective offspring (unpublished results). It is possible that SU's offspring may have learnt to be tolerant by observing her behavioural interactions with other individuals, although it is not possible to completely rule out the contributions of a suitable temperament or a genetic predisposition to display preferentially affiliative behaviour among the members of this particular matriline.

\subsection{Group-specific behaviour}

Marked differences in behavioural patterns between stable social primate groups have largely been studied using an ethnographic approach (for example, Whiten et al 1999). Primate groups, however, may occupy distinct habitats and may often be genetically unrelated, leading to criticism that such ethnographic patterns may be the results of individual learning in different environments, or may have been caused by genetic differences (Galef 1992). Two instances of distinct group-specific behavioural pat- 
terns, observed in this study on bonnet macaques, are briefly described here (see also table 1) and the frequency of their occurrence listed in table 2.

5.3a Foraging style: Individual macaques belonging to two troops - GK1 and GK2 - in Population II showed a striking difference in the manner in which they fed on buds, flowers or fruits that were clustered together on a branch or inflorescence. During most foraging sessions over the entire observation period (August 1994 to September 1995), all the nine adult females and five adult males of troop GK2 broke off twigs, branches or inflorescences - one at a time - and fed on the buds, flowers or fruits sequentially, while holding the branch or inflorescence firmly by hand. Members of troop GK1 (11 adult females and nine adult males), on the other hand, moved continuously over the branches of the tree, choosing and picking up buds, flowers or fruits randomly across branches or inflorescences; only rarely did an individual break off a particular inflorescence or branch (table 2).

5.3b Branch shaking: Male bonnet macaques, particularly dominant males, regularly shake branches during aggressive encounters with other individuals or as a display behaviour. This behaviour is much less common among adult females. The six most dominant of the nine adult females of troop GK2, however, shook branches very frequently at human observers, particularly during the initial observations (July to September 1994), and less frequently towards other troop females during agonistic interactions over the entire observation period (July 1994 to September 1995; table 2). Remarkably, not once did any of the 11 adult females of the neighbouring troop GK1 display this behaviour under any circumstance over the entire observation period (March 1993 to September 1994). It should be noted that although these two troops of bonnet macaques in Population II displayed significant differences in group-specific behaviour patterns of foraging style and branch shaking, they shared the same habitat and frequently interacted with each other - thus ruling out environmental causation for these behavioural variants.

5.3c Female affiliative relationships: A comparison between the affiliative relationships displayed by adult females in two troops - BM15 and GK2 - in two geographically separated populations (Populations I and II respectively) during the period from 1993 to 1997 revealed striking differences that could be potentially ascribed to ecological differences in their food availability and distribution (Sinha $\mathrm{A}$, in preparation). In troop BM15, for example, where the adult females periodically foraged on limited and patchily distributed human foods and competition was strong, individuals directed their allogrooming up the dominance hierarchy with subordinate females grooming dominant individuals at relatively higher levels than those subordinate to them (Ram et al 2003). In troop GK2, in contrast, individual females foraged only on natural food sources, competition for resources was relatively low, and individuals were observed to preferentially allogroom those subordinate to them (Sinha 1996). What is noteworthy, however, is that the patterns of allogrooming between the present-day adult females of troop GK2 has remained strikingly similar to that originally observed in the troop although there has been a significant change in its feeding ecology over this period-from complete natural foraging to a regime where the troop now largely feeds on provisioned human foods (Chatterjee 2003; Sinha A, in preparation). Such a longitudinal maintenance of similar behavioural patterns in this troop is reminiscent of the intergenerational transmission of maternal social networks observed in other macaques and could also imply the involvement of mother-daughter transmission mechanisms in this species (reviewed in Chauvin and Berman 2004).

\section{Cultural evolution in bonnet macaques}

A particularly remarkable example of a stable social tradition in many female-bonded cercopithecine primates,

Table 2. Frequency of group-specific behaviours displayed by bonnet macaques in Population I.

\begin{tabular}{llcc}
\hline Behaviour & \multicolumn{1}{c}{ Phenotype } & \multicolumn{2}{c}{ Troop } \\
\hline Foraging style & & GK1 & GK2 \\
\cline { 2 - 4 } & Branch or inflorescence breaking & $0 \cdot 070 \pm 0 \cdot 025$ & $0 \cdot 950 \pm 0 \cdot 475$ \\
& Bud or flower picking & $0 \cdot 875 \pm 0 \cdot 150$ & $1 \cdot 450 \pm 0 \cdot 150$ \\
Branch shaking & 0 & $0 \cdot 175 \pm 0 \cdot 125$ \\
\hline
\end{tabular}

Frequency of the observed behaviours has been represented as mean \pm SE of behavioural bouts/h/individual for foraging style and events/h/individual for branch shaking. 
including bonnet macaques, is that of the cultural inheritance of rank positions in the dominance hierarchy that operates among females. Thus, females of these species not only tend to maintain stable dominance ranks over their lifetimes (Koyama 1967; Sade 1972; Hausfater et al 1982), but adult daughters acquire ranks in the hierarchy just below their mothers and above their older sisters ('youngest ascendancy'; de Waal 1977; Datta 1983; Sinha 2001). Interestingly, longitudinal observations of adopted infants who eventually acquire their foster mothers' ranks, suggest that the status a female attains is not dependent on genetic factors, but rather on social influences during ontogeny (Itani 1959; Bernstein 1969).

In general, females appear to be dependent on the support of other dominant individuals, including their mothers, for the acquisition and maintenance of their ranks, intervention, rather than solicited support, is the main mechanism bringing about rank changes across matrilines (even though this is rare), and low rates of intervention are sufficient for the process of rank acquisition and its maintenance (Chapais 1988a,b; reviewed in Hill and Okayasu 1996).

Observational studies of macaque infants suggest that some of the ways in which they learn to select appropriate targets for aggression during acquisition of dominance ranks may involve social transmission (Berman 1980; Chapais and Gauthier 1993). Thus, although these infants develop 'dependent ranks' that correlate with those of their mothers simply because the latter actively control the outcome of their infants' agonistic interactions with all lower-ranking individuals including their older daughters, infants may also learn whom they can threaten and to whom they must defer by directly observing the agonistic interactions of their mothers.

It is indeed remarkable that such a cultural determination of rank positions in the female dominance hierarchy is in direct contrast to the development of the male dominance hierarchy in these very species, including bonnet macaques. For males, age, physical body condition, general competitive abilities, manipulative skills (leading to an ability to form effective coalitions) and perhaps, a suitable temperament are the best predictors of dominance ranks. All of these are traits that can clearly be under direct genetic control and on which natural selection can easily act.

What is, however, noteworthy among these female primates is that notwithstanding the fact that there are obviously no clear genetic or physiological determinants of their dominance ranks, high rank among females in some species do bring increased access to limited resources and ultimately increase reproductive success (Silk 1987). Although it is reasonable to hypothesise that the propensity to be assertive in situations involving acquisition of dominance ranks may be in part a product of natural selection, it is far from clear how a traditional system of maternal rank transmission per se could have been selected.

Moreover, individual females of some species often display strongly correlated physiological and behavioural traits associated with their respective positions in the rank hierarchy. A striking example of this is the skewing of birth sex ratios toward either sex exhibited by highranking macaque mothers in certain situations (bonnet macaques: Silk et al 1981; Silk 1988; rhesus macaques: Simpson and Simpson 1982; Meikle et al 1984; Nevison et al 1996; Nevison 1997; longtailed macaques: van Schaik et al 1989; Barbary macaques: Paul and Kuester 1990). Although specific proximate mechanisms underlying skews in infant sex ratio either in captive bonnet macaques (Silk et al 1981; Silk 1988) or in other primates (see Packer et al 2001) remain unknown, it is indeed remarkable that they may have been selected for to enable the facultative determination of infant sex among female primates in response to appropriate environmental or social stimuli (but see Brown and Silk 2002).

Female bonnet macaques are also able to display different social strategies toward other members of the troop in accordance with their own ranks in the dominance hierarchy (Sinha 1996; Ram et al 2003). Such an ability could arise in these females if, as mentioned earlier, they are able to learn by observation from their mothers the nature of social relationships they must develop with other females appropriate to their own positions in the hierarchy. Moreover, both the skewing of infant sex ratios and the adoption of variable social strategies must involve, in addition, the evaluation and recognition by each female of her own unique rank in the dominance hierarchy - a cognitive ability that has now clearly been demonstrated in bonnet macaques (Sinha 1998, 2003) but hardly investigated in other species. Although we are still far from understanding the exact mechanisms by which such physiological and behavioural traits become strongly correlated to the dominance status of their bearers, this must involve a process of cultural selection that will ensure the repeated appearance of these traits and their operation in females who differ from each other only in the socially transmitted inheritance of their dominance ranks.

\section{Gene-culture coevolution}

An important issue that has attracted attention but needs to be understood in much greater depth concerns the interconnections between the basic genetic inheritance system and the evolved behavioural transmission system the linkages between underlying genes and the overtly manifest social traditions or culture. In general, variation 
in socially acquired and transmitted behaviours is unlikely to be under the direct control of a single or a few genes (as are more rigid developmental variations including polyphenism) since individuals who are closely related genetically, or sometimes even genetically identical, can have and pass on very different behaviours; this is also true for social traditions that such behaviours may have generated. Social learning, however, can have profound effects on the evolution of behaviour, and current interpretations of evolutionary change need to take this into account. For example, the inheritance of a new pattern of behaviour in a population may not necessarily be due to genetic variations. Mechanisms of behavioural transmission and the possibility of a new social tradition being established should be seriously considered.

It is important to note that the assumption of behavioural inheritance can sometimes provide important alternative or complementary interpretations of known patterns of behaviour. A striking example of this has been the work of Jablonka and her colleagues who showed, for instance, that, although genetically unfavourable, adoption can spread and be maintained within animal populations through the social learning of certain parenting styles (Avital and Jablonka 1994, 1996; Avital et al 1998).

Sexual imprinting and other mechanisms of social learning can also have far-reaching demographic consequences, and, in some cases, could conceivably even initiate processes of speciation (Baird et al 1992; Hoelzel et al 1998). Recently-acquired practices and traditions, such as the pine cone-stripping described above, could precede genetic adaptations, and exert persistent directional selection for genetic variations that are congruent with the new pattern of behaviour-primarily because such traditions arise within and lead to the perpetuation of the same selective regime. To return to our previous example, the continued use of pine seeds as a major source of food by the Israeli black rats should thus provide a selective advantage to any genetic variation that improves the finding, processing, and digestion of these seeds. Moreover, selection for the ability to learn this particular behaviour pattern more efficiently and rapidly may eventually lead to it becoming dependent on fewer learning trials, or even on no learning trials at all - resulting ultimately in behavioural canalization (Jablonka et al 1998). A closer examination of the genetic mechanisms underlying such a process (variously described as canalization, genetic assimilation or the Baldwin effect), however, lies outside the scope of this paper (but see Waddington 1957; Hardy 1965; Nanjundiah 2003; Bateson 2004 for detailed discussions).

Of particular relevance to traditions or cultures that arise in primate societies is the fact that a behaviour pattern which becomes traditional through social learning is often more enduring than that acquired by individual learning; this is because it emerges from a self-sustaining social structure, and may continue to exist and be transmitted even when the environmental conditions that first initiated it in a particular individual have changed. Behavioural inheritance of this kind can also impact very strongly on the structure and dynamics of primate social systems such as the one exemplified by bonnet macaques. Since primates lead extremely complex social lives (see, for example, Sinha 2001) and may develop alternative behavioural strategies, either, as described above, through phenotypic flexibility or through the development of socially transmitted cultural traditions, it must be assumed that these behaviours ultimately contribute to the inclusive fitness of the individual and hence, are likely to interconnect with the underlying genetics of the individual or even the species.

What behavioural and life-history characteristics of bonnet macaques could facilitate the appearance and establishment of social traditions in this species and also play a role in potential gene-culture coevolution in this species over time?

Bonnet macaques possess several biological attributes that Roper (1986) suggests favour social learning in a species: a complex social organization (Sinha 2001), long lifetimes (over 20 years), advanced cognitive abilities (Sinha 1997, 1998, 2003) and relatively prolonged parental care. Russell and Russell (1990) have pointed out the link between maternal care and cultural transmission in early humans and other primates. The stable matrilineal social structure (with its concomitant cultural determination of dominance ranks) and the relatively long period of juvenile dependence, presented by bonnet macaques, may thus both permit stable transmission of behavioural traits and patterns during maternal care. Yet another biological attribute that may have a complex relationship with cultural traditions is menopause (Norris and Pryor 1991; Boran and Heimlich 1999). Many bonnet macaque females may live years after the birth of their last offspring. It is conceivable that such an extended postmenopausal life of females may allow for the transfer of environmental information across generations to increase the fitness of their offspring and relatives. If true, this would suggest that menopause and post-reproductive longevity are highly adaptive in promoting culture and may have been, in turn, selected for during evolution by within-group cultural processes. Support for such a link between post-menopausal survival and social traditions comes from our observations in the two populations of bonnet macaques (Populations I and II, table 1) wherein the direction of troop movement in most troops is usually and fairly consistently determined by the oldest males or females in the troop. This behavioural tradition is rather specific in that it is only the direction in which the troop will move - and not the time at which this movement will 
commence - that appears to be decided upon by the oldest individuals. Although it is far from clear how the troop - as a group - arrives at such a consensus and exactly how the responsibility is, in time, passed on to the oldest members of the troop, it may involve some recognition of one's own age and those of the other members of a social group - perhaps by a process akin to that involved in the evaluation of one's and other's positions in the dominance hierarchy (Sinha 1998, 2003).

Finally, although there probably is a minimum cognitive ability required for social learning, the ability of an individual or a group to develop and perpetuate social traditions also perhaps depends fairly significantly on the ecological milieu in which the species finds itself. Bonnet macaques are 'weed macaques' (Richard et al 1989) - a term testifying to their remarkable success in most ecological environments ranging from montane evergreen forests to moist deciduous forests and dry scrub jungles, and including degraded ecological sites close to human habitations. Such an achievement may have been possible only due to the tremendous variability in life-history strategies that populations of this species are genetically or behaviourally predisposed to evolve in different ecological settings (see also Singh and Sinha 2004) and this success, in turn, may have promoted the further development and consolidation of appropriate behavioural traditions.

\section{Acknowledgements}

I sincerely thank Kakoli Mukhopadhyay and Anirban Datta-Roy for their assistance in fieldwork, T R Shankar Raman for provocative discussions, and the Karnataka and Tamil Nadu Forest Departments for permission to work in the Bandipur National Park and the Mudumalai Wildlife Sanctuary respectively. I also express my gratitude to Patrick Bateson, Vidyanand Nanjundiah and an anonymous referee for constructive comments on an earlier version of this paper. This study was made possible by two Individual Research Grants (Gr6416 and Gr7816) from the Wenner-Gren Foundation for Anthropological Research, New York. A part of this work has earlier been presented at the Discussion Meeting on Genetic Determinism held at Pelling (Sikkim) in December 2003.

\section{References}

Aisner R and Terkel J 1992 Ontogeny of pine cone opening behaviour in the black rat, Rattus rattus; Anim. Behav. 44 327-336

Avital E and Jablonka E 1994 Social learning and the evolution of behaviour; Anim. Behav. 48 1195-1199

Avital E and Jablonka E 1996 Adoption, memes and the Oedipus complex: a reply to Hansen; Anim. Behav. 51 476-477
Avital E and Jablonka E 2000 Animal traditions: behavioural inheritance in evolution (Cambridge: Cambridge University Press)

Avital E, Jablonka E and Lachmann M 1998 Adopting adoption; Anim. Behav. 55 1451-1459

Baird R W, Abrams P A and Dill L M 1992 Possible indirect interactions between transient and resident killer whales: implications for the evolution of foraging specializations in the genus Orcinus; Oecologia 89 125-132

Bateson P 2003 The promise of behavioural biology; Anim. Behav. 65 11-17

Bateson P 2004 The active role of behaviour in evolution; Biol. Philos. 19 283-298

Berman C M 1980 Early agonistic experience and rank acquisition among free-ranging infant rhesus monkeys; Int. J. Primatol. 1 153-170

Bernstein I S 1969 Stability of the status hierarchy in a pigtail monkey group (Macaca nemestrina); Anim. Behav. 17 452458

Boran J R and Heimlich S L 1999 Social learning in cetaceans: hunting, hearing and hierarchies; in Mammalian social learning: comparative and ecological perspectives (eds) $\mathrm{H} \mathrm{O} \mathrm{Box}$ and K R Gibson (Cambridge: Cambridge University Press) pp 282-307

Boyd R and Richerson P J 1985 Culture and the evolutionary process (Chicago: Chicago University Press)

Brown G R and Silk J B 2002 Reconsidering the null hypothesis: is maternal rank associated with birth sex ratios in primate groups?; Proc. Natl. Acad. Sci. USA 99 11252-11255

Byrne R W, Barnard P J, Davidson I, Janik V M, McGrew W C, Miklósi Á and Wiessner P 2004 Understanding culture across species; Trends Cogn. Sci. 8 341-346

Cavalli-Sforza L L and Feldman M W 1981 Cultural transmission and evolution: a quantitative approach (Princeton: Princeton University Press)

Chapais B 1988a Experimental matrilineal inheritance of rank in female Japanese macaques; Anim. Behav. 36 1025-1037

Chapais B 1988b Rank maintenance in female Japanese macaques: experimental evidence for social dependency; Behaviour 104 41-59

Chapais B and Gauthier C 1993 Early agonistic experience and the onset of matrilineal rank acquisition in Japanese macaques; in Juvenile primates: life history, development, and behaviour (eds) M E Pereira and L J Fairbanks (New York: Oxford University Press) pp 245-258

Chatterjee M 2003 Social behaviour of wild bonnet macaques: affiliative and agonistic behaviour of adult females (Unpublished Report, submitted to Indian Academy of Sciences, Bangalore)

Chauvin C and Berman C M 2004 Intergenerational transmission of behavior; in Macaque societies: a model for the study of social organization (eds) B Thierry, M Singh and W Kaumanns (Cambridge: Cambridge University Press) pp 209-234

Danks H V 1999 Life cycles in polar arthropods - flexible or programmed?; Eur. J. Entomol. 96 83-103

Datta S B 1983 Relative power and acquisition of rank; in Primate social relationships: an integrated approach (ed.) R A Hinde (Oxford: Blackwell) pp 93-103

de Waal F B M 1977 The organization of agonistic relations within two captive groups of Java-monkeys (Macaca fascicularis); Z. Tierpsychol. 44 225-282

Drapier M and Thierry B 2002 Social transmission of feeding techniques in Tonkean macaques?; Int. J. Primatol. 23 105122 
Dugatkin L A 1996 Interface between culturally based preferences and genetic preferences: female mate choice in Poecilia reticulata; Proc. Natl. Acad. Sci. USA 93 2770-2773

Enggist-Dueblin P and Pfister U 2002 Cultural transmission of vocalizations in ravens; Anim. Behav. 64 831-841

Feldman M W and Laland K N 1996 Gene-culture coevolutionary theory; Trends Ecol. Evol. 11 453-457

Fisher J and Hinde R A 1950 The opening of milk bottles by birds; Brit. Birds 42 347-357

Fisher J and Hinde R A 1952 Further, observations on the opening of milk bottles by birds; Brit. Birds 44 393-396

Galef B G Jr 1992 The question of animal culture; Hum. Nature 3 157-178

Gordon D M 1992 Phenotypic plasticity; in Keywords in evolutionary biology (eds) E F Keller and E A Lloyd (Cambridge, Mass: Harvard University Press) pp 255-262

Hardy A 1965 The living stream (London: Collins)

Hausfater G, Altmann J and Altmann S 1982 Long-term consistency of dominance relations among female baboons (Papio cynocephalus); Science 217 752-755

Heyes C M and Galef B G (eds) 1996 Social learning in animals: the roots of culture (San Diego: Academic Press)

Hill D A and Okayasu N 1996 Determinants of dominance among female macaques: nepotism, demography and danger; in Evolution and ecology of macaque societies (eds) J E Fa and D G Lindburg (Cambridge: Cambridge University Press) pp 459-472

Ho M W and Saunders P T (eds) 1984 Beyond neo-Darwinism. Introduction to the new evolutionary paradigm (London: Academic Press)

Hoelzel A R, Dahlheim M and Stern S J 1998 Low genetic variation among killer whales (Orcinus orca) in the Eastern North Pacific and genetic differentiation between foraging specialists; J. Hered. 89 121-128

Hook M A, Lambeth S P, Perlman J E, Stavisky R, Bloomsmith M A and Schapiro S J 2002 Inter-group variation in abnormal behaviour in chimpanzees (Pan troglodytes) and rhesus macaques (Macaca mulatta); Appl. Anim. Behav. Sci. 76 $165-176$

Itani J 1959 Paternal care in the wild Japanese monkey, Macaca fuscata fuscata; Primates 2 61-94

Jablonka E 1994 Inheritance systems and the evolution of new levels of individuality; J. Theor. Biol. 170 301-309

Jablonka E and Szathmáry E 1995 The evolution of information storage and heredity; Trends Ecol. Evol. 10 206-211

Jablonka E and Lamb M J 1989 The inheritance of acquired epigenetic variations; J. Theor. Biol. 139 69-83

Jablonka E and Lamb M J 1998 Epigenetic inheritance in evolution; J. Evol. Biol. 11 159-183

Jablonka E and Lamb M J 2002 The changing concepts of epigenetics; Ann. N.Y. Acad. Sci. 981 82-96

Jablonka E, Lamb M J and Avital E 1998 'Lamarckian' mechanisms in Darwinian evolution; Trends Ecol. Evol. 13 206-210

Kawai M 1965 Newly-acquired pre-cultural behaviour of the natural troop of Japanese monkeys on Koshima islet; Primates 6 1-30

Kawai M, Watanabe K and Mori A 1992 Pre-cultural behaviors observed in free-ranging Japanese monkeys on Koshima Islet over the past 25 years; Primate Rep. 32 143-153

Kawamura S 1959 The process of sub-culture propagation among Japanese macaques; Primates 2 43-60

Kingsolver J G and Huey R B 1998 Evolutionary analyses of morphological and physiological plasticity in thermally variable environments; Am. Zool. 38 545-560
Koyama N 1967 On dominance rank and kinship of a wild Japanese monkey troop in Arashiyama; Primates 8 189-216

Kummer H and Goodall J 1985 Conditions of innovative behaviour in primates; Philos. Trans. R.. Soc. London B. 308 203-214

Kuper A 1999 Culture: the anthropologist's account (Cambridge, Mass: Harvard University Press)

Laland K N and Hoppit W 2003 Do animals have culture?; Evol. Anthropol. 12 150-159

Maynard Smith J and Szathmáry E 1995 The major transitions in evolution (London: Collins)

Mayr E 1963 Animal species and evolution (Cambridge, Mass: Harvard University Press)

Mayr E 1969 Principles of systematic zoology (New York: McGraw-Hill)

Meikle D B, Tilford B L and Vessey S H 1984 Dominance rank, secondary ratio and reproduction of offspring in polygynous primates; Am. Nat. 124 173-188

Montgomerie R 2001 Dirty ptarmigan: behavioral modification of conspicuous male plumage; Behav. Ecol. 12 429-438

Nanjundiah V 2003 Phenotypic plasticity and evolution by genetic assimilation; in Origination of organismal form (eds) G B Muller and S A Newman (Cambridge, Mass: MIT Press) pp 245-263

Nevison C M 1997 Social rank and birth sex ratios in rhesus monkeys (Macaca mulatta); Appl. Anim.Behav.Sci. 51 285291

Nevison C M, Rayment F D G and Simpson M J A 1996 Birth sex ratio and maternal social rank in a captive colony of rhesus monkeys (Macaca mulatta); Am. J. Primatol. 39 123-138

Norris K S and Pryor K 1991 Some thoughts on grandmothers; in Dolphin societies: discoveries and puzzles (eds) K Pryor and K S Norris (Berkeley: University of California Press) pp 287-289

Packer C, Collins D A and Eberly L E 2001 Problems with primate sex ratios; Proc. R. Soc. London B 355 1627-1635

Padilla D K and Adolph S C 1996 Plastic inducible morphologies are not always adaptive: the importance of time delays in a stochastic environment; Evol. Ecol. 10 105-107

Paul A and Kuester J 1990 Adaptive significance of sex ratio adjustment in semi-free-ranging Barbary macaques (Macaca sylvanus) at Salem; Behav. Ecol. Sociobiol. 27 287-293

Pigliucci M 2001 Phenotypic plasticity (Baltimore: Johns Hopkins University Press)

Piersma T and Drent J 2003 Phenotypic flexibility and the evolution of organismal design; Trends Ecol. Evol. 18 228-233

Rakyan V K, Preis J, Morgan H D and Whitelaw E 2001 The marks, mechanisms and memory of epigenetic states in mammals; Biochem. J. 356 1-10

Ram S, Venkatachalam S and Sinha A 2003 Changing social strategies of wild female bonnet macaques during natural foraging and on provisioning; Curr. Sci. 84 780-790

Rendell L and Whitehead H 2001 Culture in whales and dolphins; Behav. Brain Sci. 24 309-382

Reznick D and Travis J 1996 The empirical study of adaptation in natural populations; in Adaptation (eds) M Rose and G V Lauder (New York: Academic Press) pp 243-289

Richard A F, Goldstein S J and Dewar R E 1989 The evolutionary implications of macaque feeding ecology; Int. J. Primatol. 10 569-591

Rollo C D 1994 Phenotypes: their epigenetics, ecology and evolution (London: Chapman and Hall)

Roper T J 1986 Cultural evolution of feeding behaviour in animals; Sci. Prog. 70 571-583 
Russell C and Russell W M S 1990 Cultural evolution of behaviour; Neth. J. Zool. 40 745-762

Sade D S 1972 A longitudinal study of social behaviour of rhesus monkeys; in Functional and evolutionary biology of primates (ed.) R Tuttle (Chicago: Aldine) pp 378-398

Schlichting C D and Pigliucci M 1998 Phenotypic evolution: a reaction norm perspective (Sunderland, Mass: Sinauer)

Shapiro A M 1976 Seasonal polyphenism; Evol. Biol. 9 259333

Silk J B 1987 Social behaviour in evolutionary perspective; in Primate societies (eds) B B Smuts, D L Cheney, R M Seyfarth, R W Wrangham and T T Struhsaker (Chicago: University of Chicago Press) pp 318-329

Silk J B 1988 Maternal investment in captive bonnet macaques (Macaca radiata); Am. Nat. 132 1-19

Silk J B, Clark-Wheatley C B, Rodman P S and Samuels A 1981 Differential reproductive success and facultative adjustment of sex ratios among captive female bonnet macaques (Macaca radiata); Anim. Behav. 29 1106-1120

Simpson M J A and Simpson A E 1982 Birth sex ratios and social rank in rhesus macaque mothers; Nature (London) $\mathbf{3 0 0}$ 440-441

Singh M and Sinha A 2004 Life-history traits (in macaques): Ecological adaptations or phylogenetic relics?; in Macaque societies: a model for the study of social organization (eds) B Thierry, M Singh and W Kaumanns (Cambridge: Cambridge University Press) pp 80-83

Sinha A 1996 A new twist in an old macaque tale: allogrooming relationships among wild female bonnet macaques; in Abstracts of the Sixteenth Congress of the International Primatological Society (Madison: Wisconsin Regional Primate Research Center) p 286

Sinha A 1997 Complex tool manufacture by a wild bonnet macaque, Macaca radiata; Folia Primatol. 68 23-25

Sinha A 1998 Knowledge acquired and decisions made: triadic interactions during allogrooming in wild bonnet macaques, Macaca radiata; Philos. Trans. R. Soc. London B 253 619631

Sinha A 2001 The monkey in the town's commons: a natural history of the Indian bonnet macaque (NIAS Report R 2-01) (Bangalore: National Institute of Advanced Studies)

Sinha A 2003 A beautiful mind: Attribution and intentionality in wild bonnet macaques; Curr. Sci. 85 1021-1030

Sinha A, Mukhopadhyay K and Datta-Roy A 2003 Evolution of unimale social organisation in bonnet macaques (Macaca radiata radiata); in Proceedings of the Twenty-eighth Conference of the Ethological Society of India (eds) R Annamalai, M Narayanan and J Vanitharani (Tirunelveli: Department of Zoology, Sarah Tucker College and Tamil Nadu Forest Department, Kalakad-Mundanthurai Tiger Reserve) pp 110 115

Sinha A, Mukhopadhyay K, Datta-Roy A and Ram S 2005 Ecology proposes, behaviour disposes: ecological variability in social organisation and male behavioural strategies among wild bonnet macaques; Curr. Sci. (in press)

Smuts B, Cheney D L, Seyfarth R M, Wrangham R W and
Struhsaker T T (eds) 1987 Primate societies (Chicago: University of Chicago Press)

Sultan S E 1995 Phenotypic plasticity and plant adaptation; Acta Bot. Neerl. 44 363-383

Stearns S 1989 The evolutionary significance of phenotypic plasticity; Bioscience 39 436-445

Terkel J 1996 Cultural transmission of feeding behaviour in the black rat (Rattus rattus); in Social learning in animals: the roots of culture (eds) C M Heyes and B G Galef (San Diego: Academic Press) pp 17-47

Tomasello M 1990 Cultural transmission in chimpanzee tool use and signaling; in Language and intelligence in monkeys and apes (eds) S T Parker and $\mathrm{K} \mathrm{R}$ Gibson (Cambridge: Cambridge University Press) pp 274-311

Tomasello M 1996 Do apes ape?; in Social learning in animals: the roots of culture (eds) C M Heyes and B G Galef Jr (San Diego: Academic Press) pp 319-346

Tomasello M, Davis-Dasilva M, Carnak L and Bard K 1987 Observational learning of tool-use by young chimpanzees; Hum. Evol. 2 175-183

Tomasello M, Kruger A C and Ratner H H 1993 Cultural learning; Behav. Brain Sci. 16 495-511

Travis J 1994 Evaluating the adaptive role of morphological plasticity; in Ecological morphology: integrative organismal biology (eds) P C Wainwright and S M Reilly (Chicago: Chicago University Press) pp 99-122

Travis J, McManus M G and Baer C F 1999 Sources of variation in physiological phenotypes and their evolutionary significance; Am. Zool. 39 422-433

van Schaik C P, Netto W J, van Amerongen A J J and Westland H 1989 Social rank and sex ratio of captive long-tailed macaque females (Macaca fascicularis); Am. J. Primatol. 19 147161

van Schaik C P, Ancrenaz M, Borgen G, Galdikas B, Knott C D, Singleton I, Suzuki A, Utami S S and Merrill M 2003 Orangutan cultures and the evolution of material culture; Science 299 102-105

Via S, Gomulkiewicz R, De Jong G, Scheiner S M, Schlichting C D and Van Tienderen P H 1995 Adaptive phenotypic plasticity: consensus and controversy; Trends Ecol. Evol. 19212 217

Waddington C H 1957 The strategy of the genes (London: Allen and Unwin)

West-Eberhard M J 1989 Phenotypic plasticity and the origins of diversity; Annu. Rev. Ecol. Syst. 20 249-278

Whiten A 2000 Primate culture and social learning; Cognitive Sci. 24 477-508

Whiten A and Ham R 1992 On the nature and evolution of imitation in the animal kingdom: reappraisal of a century of research; in Advances in the study of behaviour (eds) P J B Slater, J S Rosenblatt, C Beer and M Milinski (San Diego: Academic Press) pp 239-283

Whiten A, Goodall J, McGrew W C, Nishida T, Reynolds V, Sugiyama Y, Tutin C E G, Wrangham R W and Boesch C 1999 Cultures in chimpanzees; Nature (London) 399682 685

$e$ Publication: 11 March 2005 